\title{
A NONLINEAR ERGODIC THEOREM FOR DISCRETE SYSTEMS
}

\author{
K. M. Crowe \\ Biometrics Unit \\ Cornell University \\ Ithaca, NY 14853
}

BUJ-1176-M

Seotember 1992

Supported in the Program in Applied Mathematics at the University of Arizona by the Applied Mathematics and Population Biology/Ecology Divisions of the National Science Foundation under grant No. DMS-8902508. Supported at Cornell University by the U.S. Army Research Office through the Mathematical Sciences Institute, Contract DAAL03-91-C-0027, and by the National Science Foundation through the Biometrics Unit, grant No. DEB9253570 


\begin{abstract}
A theorem concerning the asymptotic behavior of a general class of systems of difference equations is proven. This theorem guarantees the existence of a stable normalized distribution vector, and enables the constructing of a scalar limiting equation for an aggregate variable. This theory is illustrated using an example from population dynamics, a nonlinear size-structured model of competition for a dynamically modeled limiting resource.
\end{abstract}

Key words: Nonlinear Ergodic Theorem, size-structured competition model, limiting equation 


\section{Introduction}

We consider the discrete nonlinear system

$$
\vec{x}(t)=\mathcal{P}(t) \vec{x}(t)
$$

where $\vec{x}(t)=\operatorname{col}\left(x_{i}(t)\right)_{i=1}^{m}$ and $\mathcal{P}(t)$ is a nonnegative $m \times m$ matrix. We assume that $\mathcal{P}$ can be written in the form

$$
\mathcal{P}(t)=a_{0}(t) \mathcal{I}+a_{1}(t) \mathcal{L}+\cdots+a_{r}(t) \mathcal{L}^{r}
$$

where $\mathcal{L}$ is a constant nonnegative matrix and the $a_{i}(t)$ are scalar functions.

Nonnegative matrices play an important role in many fields of applied mathematics, and since the publication of the work of Perron and Frobenius, the theory of nonnegative matrices has been applied to such diverse areas as demography, economics, genetics, population dynamics, and probability theory. In this paper we consider the preceding general class of nonlinear matrix difference equations and obtain a result which describes the asymptotic behavior of the normalized distribution vector corresponding to $\vec{x}(t)$. We illustrate the usefulness of this result by considering a size-structured competition model in which the transition probabilities and birth functionals are dependent on various individual-level physiological parameters. This produces a fairly realistic but somewhat intractable matrix difference equation of the form (1). Using the theory described here, we are able to study an 
asymptotic scalar difference equation for total population size without losing information about the dependence of our results on the individual level parameters.

In $\S 2$ we state and prove the main result, an extension of work done by Cushing [6] and Impagliazzo [11] under more restrictive assumptions. In addition, we discuss the implications of this result in the study of the system (1). In $\S 3$ we describe in detail an application of this theory to a class of models of size-structured competition for a limited resource and explore numerically a specific example which illustrates the significance of the theory. Finally, in $\S 4$, we discuss the significance and potential usefulness of this result in the field of population dynamics.

\section{Nonlinear Ergodic Theorem}

Consider the system of difference equations given by

$$
\begin{gathered}
\vec{x}(t+1)=\mathcal{P}(t) \vec{x}(t) \\
\mathcal{P}(t)=a_{0}(t) \mathcal{I}+a_{1}(t) \mathcal{L}+a_{2}(t) \mathcal{L}^{2}+\cdots+a_{r}(t) \mathcal{L}^{r}
\end{gathered}
$$

where $\mathcal{I}$ is the $m \times m$ identity matrix and $\mathcal{L}$ is a constant nonnegative $m \times m$ matrix. Here the dependence of the $a_{i}(t)$ and on time may be explicit and/or implicit via dependence (either linear or nonlinear) on the $x_{i}(t)$. In addition, let $\vec{w}$ be a given vector in $\Re^{m}$ with $\vec{w} \geq \overrightarrow{0}$ and $\vec{w} \neq \overrightarrow{0}$ and define

$$
P(t)=\vec{w}^{T} \vec{x}(t)=\sum_{j=1}^{m} w_{j} x_{j}(t)
$$


the inner product of $\vec{w}$ and $\vec{x}(t)$. Then we can define the normalized distribution corresponding to $\vec{x}(t)$ by

$$
\vec{\eta}(t)=\frac{\vec{x}(t)}{P(t)} .
$$

We then have the following result.

Theorem 1 (Nonlinear Ergodic Theorem) Assume that the following two hypotheses hold.

H1 There exist constants $a_{0}$ and $b_{0}$ such that $0 \leq a_{0}(t) \leq a_{0}$ and $0<b_{0} \leq a_{i}(t), i=1, \ldots, r$ for all $t=0,1,2, \ldots$.

H2 $\mathcal{L}$ has a strictly dominant positive simple eigenvalue $\lambda^{+}>0$ with an associated positive eigenvector $\vec{v}^{+}$which is normalized so that $\vec{w}^{T} \vec{v}^{+}=1$.

Also assume that $\vec{x}(t) \geq \overrightarrow{0}$ is a solution of Equations (3) such that $\vec{x}(0) \geq \overrightarrow{0}$, $\vec{x}(0) \neq \overrightarrow{0}$ and $P(t)>0$ for $t \geq 0$. Then $\vec{\eta}(t) \rightarrow \vec{v}^{+}$as $t \rightarrow+\infty$.

Proof: Combining Equations (3), we obtain

$$
\vec{x}(t+1)=\left(a_{0}(t) \mathcal{I}+\cdots+a_{r}(t) \mathcal{L}^{r}\right) \vec{x}(t) .
$$

Assume that $\mathcal{L}$ has eigenvalues $\lambda_{1}=\lambda^{+}, \lambda_{2}, \ldots \lambda_{q}$, each with multiplicity $m(i)$, so that $m(1)=1$ and $\sum_{i=1}^{q} m(i)=m$. Then $\lambda_{1}$ has corresponding 
eigenvector $\vec{v}^{+}$, and $\lambda_{i}$ has eigenvectors (and/or generalized eigenvectors) $\vec{v}_{i, 1}, \vec{v}_{i, 2}, \ldots, \vec{v}_{i, m(i)}$ such that the set

$$
\left\{\vec{v}^{+}, \vec{v}_{2,1}, \ldots, \vec{v}_{2, m(2)}, \vec{v}_{3,1}, \ldots, \vec{v}_{3, m(3)}, \ldots, \vec{v}_{q, 1}, \vec{v}_{q, m(q)}\right\}
$$

is linearly independent and thus spans $\Re^{m}$.

The coefficient matrix $a_{0}(t) \mathcal{I}+a_{1}(t) \mathcal{L}+\cdots+a_{r}(t) \mathcal{L}^{r}$ has eigenvalues $a_{0}(t)+a_{1}(t) \lambda_{i}+\cdots a_{r}(t) \lambda_{i}^{r}$ with the same set, $Q$, of eigenvectors as given above. Then for each $\tau \geq 0$ there is a Jordan matrix $\mathcal{J}(\tau)$ similar to $a_{0}(\tau) \mathcal{I}+$ $a_{1}(\tau) \mathcal{L}+\cdots a_{r}(\tau) \mathcal{L}^{r}\left(\right.$ i.e. $a_{0}(\tau) \mathcal{I}+a_{1}(\tau) \mathcal{L}+\cdots+a_{r}(\tau) \mathcal{L}^{r}=\mathcal{T} \mathcal{J}(\tau) \mathcal{T}^{-1}$ for some nonsingular matrix $\mathcal{T}$ ) of the form

$$
\mathcal{J}(\tau)=\operatorname{diag}\left[\mathcal{J}_{1}(\tau), \mathcal{J}_{2}(\tau), \ldots, \mathcal{J}_{q}(\tau)\right]
$$

where $\mathcal{J}_{1}(\tau)=\left[a_{0}(\tau)+a_{1}(\tau) \lambda^{+}+\cdots+a_{r}(\tau) \lambda^{+r}\right]$ and for $i=2, \ldots, q, \mathcal{J}_{i}(\tau)$ is given by

$$
\mathcal{J}_{i}(\tau)=\operatorname{diag}\left[\mathcal{J}_{i 1}(\tau), \ldots, \mathcal{J}_{i d(i)}(\tau)\right]
$$

Here $\mathcal{J}_{i j}$ is a square matrix of order $k_{i j}$ given by

$$
\mathcal{J}_{i j}(\tau)=\left[\begin{array}{cccc}
a_{0}(\tau)+\cdots+a_{r}(\tau) \lambda_{i}^{r} & & & \\
1 & \ddots & & \mathcal{O} \\
\mathcal{O} & \ddots & \ddots & \\
& & 1 & a_{0}(\tau)+\cdots+a_{r}(\tau) \lambda_{i}^{r}
\end{array}\right]
$$

where $k_{i 1} \geq k_{i 2} \geq \cdots \geq k_{i d(i)}$ and $\sum_{j=1}^{d(i)} k_{i j}=m(i)$.

Equation (4) has solution 


$$
\begin{aligned}
\vec{x}(t) & =\prod_{\tau=0}^{t-1}\left(a_{0}(\tau) \mathcal{I}+\cdots+a_{r}(\tau) \mathcal{L}^{r}\right) \vec{x}(0) \\
& =\mathcal{T}\left(\prod_{\tau=0}^{t-1} \mathcal{J}(\tau)\right) \mathcal{T}^{-1} \vec{x}(0)
\end{aligned}
$$

where

$$
\prod_{\tau=0}^{t} \mathcal{J}(\tau)=\operatorname{diag}\left[\prod_{\tau=0}^{t} \mathcal{J}_{1}(\tau), \prod_{\tau=0}^{t} \mathcal{J}_{21}(\tau), \ldots, \prod_{\tau=0}^{t} \mathcal{J}_{2 d(2)}(\tau), \ldots, \prod_{\tau=0}^{t} \mathcal{J}_{q d(q)}(\tau)\right]
$$

Let $\mathcal{J}_{i j}^{\prime}(\tau) \triangleq \frac{\mathcal{J}_{i j}(\tau)}{a_{0}(\tau)+\cdots+a_{r}(\tau) \lambda^{+\tau}}$. Then

$$
\prod_{\tau=0}^{t} \mathcal{J}_{i j}^{\prime}(\tau)=\left(\prod_{\tau=0}^{t} \frac{1}{a_{0}(\tau)+\cdots+a_{r}(\tau) \lambda^{+r}}\right) \prod_{\tau=0}^{t} \mathcal{J}_{i j}(\tau)
$$

and

$$
\prod_{\tau=0}^{t} \mathcal{J}^{\prime}(\tau)=\left(\prod_{\tau=0}^{t} \frac{1}{a_{0}(\tau)+\cdots+a_{r}(\tau) \lambda^{+r}}\right) \prod_{\tau=0}^{t} \mathcal{J}(\tau) .
$$

Note that since the first column of $\mathcal{T}$ is $\vec{v}^{+}$, we may write

$$
\mathcal{T}\left[\begin{array}{cccc}
1 & 0 & \cdots & 0 \\
0 & 0 & \cdots & 0 \\
\vdots & \vdots & \ddots & \vdots \\
0 & 0 & \cdots & 0
\end{array}\right] \mathcal{T}^{-1} \vec{x}(0)=c_{1} \vec{v}^{+}
$$

where $\vec{c}=\left(c_{1}, c_{2}, \ldots, c_{m}\right)=\mathcal{T}^{-1} \vec{x}(0)$, and hence

$$
\begin{aligned}
& \vec{x}(t)=c_{1}\left(\prod_{\tau=0}^{t-1}\left(a_{0}(\tau)+\cdots+a_{r}(\tau) \lambda^{+r}\right)\right) \vec{v}^{+} \\
& \quad+\sum_{i=2}^{q} \sum_{j=1}^{d(i)} \mathcal{T}\left[\begin{array}{cccccc}
0 & \mathcal{O} & \cdots & \mathcal{O} & \cdots & \mathcal{O} \\
\vdots & \vdots & & \vdots & & \vdots \\
\mathcal{O} & \mathcal{O} & \cdots & \prod_{\tau=0}^{t-1} \mathcal{J}_{i j}(\tau) & \cdots & \mathcal{O} \\
\vdots & \vdots & & \vdots & & \vdots \\
\mathcal{O} & \mathcal{O} & \cdots & \mathcal{O} & \cdots & \mathcal{O}
\end{array}\right] \mathcal{T}^{-1} \vec{x}(0)
\end{aligned}
$$


Next, letting

$$
\Pi_{t} \triangleq \prod_{\tau=0}^{t-1} \frac{1}{a_{0}(\tau)+\cdots+a_{r}(\tau) \lambda^{+^{r}}}
$$

and multiplying Equation (5) by $\Pi_{t}$, we obtain

$\Pi_{t} \vec{x}(t)=c_{1} \vec{v}^{+}+\sum_{i=2}^{q} \sum_{j=1}^{d(i)} \mathcal{T}\left[\begin{array}{cccccc}0 & \mathcal{O} & \cdots & \mathcal{O} & \cdots & \mathcal{O} \\ \vdots & \vdots & & \vdots & & \vdots \\ \mathcal{O} & \mathcal{O} & \cdots & \prod_{\tau=0}^{t-1} \mathcal{J}_{i j}^{\prime}(\tau) & \cdots & \mathcal{O} \\ \vdots & \vdots & & \vdots & & \vdots \\ \mathcal{O} & \mathcal{O} & \cdots & \mathcal{O} & \cdots & \mathcal{O}\end{array}\right] \mathcal{T}^{-1}\left[\begin{array}{c}c_{1} \\ \vdots \\ \vdots \\ c_{m}\end{array}\right]$

Lemma 1 For $i=2, \ldots, q$ and $j=1, \ldots, d(i)$, each element of $\prod_{\tau=0}^{t-1} \mathcal{J}_{i j}^{\prime}(\tau)$ goes to 0 as $t \rightarrow+\infty$.

Proof: First note that $\prod_{\tau=0}^{t-1} \mathcal{J}_{i j}^{\prime}(\tau)$ is a lower triangular matrix for all $i$ and $j$, so the $r c^{\text {th }}$ element of each will be 0 for $r<c$. For $r \geq c$ we can see that the $r c^{t h}$ element will be a sum of $\left(r \frac{t}{-} c\right)$ terms of the form

$$
\frac{\prod_{\tau \in \mathcal{A}}\left(a_{0}(\tau)+\cdots+a_{r}(\tau) \lambda_{i}^{r}\right)}{\prod_{\tau=0}^{t-1}\left(a_{0}(\tau)+\cdots+a_{r}(\tau) \lambda^{+^{r}}\right)}
$$

where $\mathcal{A} \subseteq\{0, \ldots, t-1\}$ with $\# \mathcal{A}=t-r+c$. Now

$$
\begin{aligned}
& \left|\frac{\prod_{\tau \in \mathcal{A}}\left(a_{0}(\tau)+\cdots+a_{r}(\tau) \lambda_{i}^{r}\right)}{\prod_{\tau=0}^{t-1}\left(a_{0}(\tau)+\cdots+a_{r}(\tau) \lambda^{+r}\right)}\right| \\
& \quad \leq \max _{\tau \in[0, t-1)}\left\{\left|\frac{a_{0}(\tau)+\cdots+a_{t}(\tau) \lambda_{i}^{r}}{a_{0}(\tau)+\cdots+a_{r}(\tau) \lambda^{+r}}\right|^{t-r+c}\left|\frac{1}{a_{0}(\tau)+\cdots+a_{r}(\tau) \lambda^{+r}}\right|^{r-c}\right\} \\
& \quad \leq \max _{\tau \in[0, t-1)}\left\{\left|\frac{\rho(\tau)+\left(\lambda_{i}+\cdots+\lambda_{i}^{r}\right)}{\rho(\tau)+\left(\lambda^{+}+\cdots+\lambda^{+r}\right)}\right|^{t-r+c}\left|\frac{1}{a_{0}(\tau)+\cdots+a_{r}(\tau) \lambda^{+r}}\right|^{r-c}\right\}
\end{aligned}
$$


where $\rho(\tau) \triangleq \frac{a_{0}(\tau)}{\max _{i=1, \ldots, r} a_{i}(\tau)}$.

It can be seen in the complex plane that $\lambda^{+}>\left|\lambda_{i}\right|$ implies that $\lambda^{+^{j}}>$ $\left|\lambda_{i}^{j}\right|$ and hence that $\frac{|x+| \lambda_{i}|+\cdots+| \lambda_{i} \Gamma^{\mid} \mid}{x+\lambda^{+}+\cdots+\lambda^{+}}<1$ for all $x>0$. Since this ratio is continuous in $x$, we can choose some $m_{i}<1$ such that for all $x \in\left[0, \rho_{0}\right]$, $0<\frac{\left.|x+| \lambda_{i}|+\cdots+| \lambda_{i}\right|^{r} \mid}{x+\lambda^{+}+\cdots+\lambda^{+}}<m_{i}$. Here $\rho_{0}$ is the upper bound of $\rho$; i.e. $\rho_{0}=\frac{a_{0}}{b_{0}}$, which exists and is finite due to the assumptions on the $a_{i}(\tau)$. Thus we obtain

$$
\mid r c^{t h} \text { element of } \prod_{\tau=0}^{t-1} \mathcal{J}_{i j}^{\prime}(\tau) \mid \leq \frac{t^{r-c}}{(r-c) !} \frac{1}{a_{0}^{r-c}} m_{i}^{t-r+c}
$$

which approaches 0 as $t \rightarrow+\infty$.

Thus by the preceding lemma, as $t \rightarrow+\infty, \vec{x}(t) \rightarrow c_{1} \Pi_{t}^{-1} \vec{v}^{+}$, and the normalized size distribution $\vec{\eta}(t)=\frac{\vec{x}(t)}{\|\vec{x}(t)\|}$ approaches $\frac{c_{1} \Pi_{t} \vec{v}^{+}}{c_{1} \Pi_{t}}=\vec{v}^{+}$as $t \rightarrow+\infty$. Note that $c_{1}>0$ since $c_{1}=\vec{w}_{1}^{T} \vec{x}(0)$ where $\vec{w}_{1}$ is the left eigenvector of $\mathcal{L}$ corresponding to $\lambda_{1}=\lambda^{+}$, a positive vector.

Before applying this theorem, a discussion of its second hypothesis is in order. It is well known from the theory of positive matrices that the dynamics of $\vec{x}(t)$ will be determined by the eigenvalue of largest magnitude of the projection matrix. If this eigenvalue has modulus greater than one then at least one solution of Equation (3) will tend to $\infty$ exponentially as $t \rightarrow$ $+\infty$, while if this eigenvalue has modulus less than one then all solutions of Equation (3) will tend exponentially to zero as $t \rightarrow+\infty$. Since the transition matrix $\mathcal{P}(t)$ is just a power series in $\mathcal{L}$, we are concerned with the eigenvalues 
of $\mathcal{L}$.

Since $\mathcal{L}$ is nonnegative, it must have a nonnegative real eigenvalue $\lambda_{1} \geq 0$ with corresponding eigenvector $\vec{v}_{1}$ which is also nonnegative, and the moduli of all other eigenvalues cannot exceed $\lambda_{1}$ (cf. Gantmacher [9]). According to Perron's theorem, if $\mathcal{L}$ is strictly positive then so is $\lambda_{1}$, and the moduli of all other eigenvalues are strictly less than $\lambda_{1}$. However, in most applications to population dynamics $\mathcal{L}$ will have some zero entries and so we must consider Frobenius' generalization to Perron's theorem in which the requirement of positivity of $\mathcal{L}$ is replaced by the requirements of nonnegativity and irreducibility of $\mathcal{L}$.

Recall that a matrix $\mathcal{L}$ is reducible if the index set $I=\{0, \ldots, m\}$ can be partitioned into two disjoint subsets $J=\left\{j_{1}, \ldots, j_{\alpha}\right\}$ and $K=\left\{k_{1}, \ldots, k_{\beta}\right\}$ such that the $j k^{\text {th }}$ element of $\mathcal{L}$ is zero for all $j \in J, k \in K$. In other words, a permutation of $\mathcal{L}$ (a permutation of rows together with the same permutation of columns) can be performed so that $\mathcal{L}$ is placed in the block form

$$
\mathcal{L}=\left[\begin{array}{ll}
\mathcal{A} & 0 \\
\mathcal{B} & \mathcal{C}
\end{array}\right]
$$

where $\mathcal{A}$ is $\alpha \times \alpha, \mathcal{B}$ is $\beta \times \alpha$, and $\mathcal{C}$ is $\beta \times \beta$. In terms of population models, this means that there exists a subset of classes which are unreachable from the remaining classes from transitions and births.

According to Frobenius' theorem, if $\mathcal{L}$ is both nonnegative and irreducible then the (real) eigenvalue $\lambda_{1}$ is positive and algebraically simple, has a corresponding positive eigenvector, and is greater than or equal to the moduli 
of all other eigenvalues of $\mathcal{L}$.

Another concept of importance is the primitivity of a matrix. The matrix $\mathcal{L}$ is primitive if $\lambda_{1}$ is strictly larger than the moduli of all the other eigenvalues. There are several tests used to determine the primitivity (or imprimitivity) of matrices. According to Gantmacher, a matrix $\mathcal{N}$ is primitive if and only if $\mathcal{N}^{n}>0$ for some finite integer $n$. In addition, it is known that a Leslie matrix is primitive if it contains two consecutive nonzero entries in its top row. The following result, applicable to the matrices considered here, is due to a comment by J. M. Cushing (private commulication).

If $\mathcal{N}$ is any nonnegative $m \times m$ matrix, it can be written as the sum of a nonnegative matrix $\mathcal{Q}$ with top row entries zero and a Leslie matrix $\mathcal{L}$. Then

$$
\mathcal{N}^{n}=\mathcal{L}^{n}+\sum \text { nonnegative matrices. }
$$

If $\mathcal{N}$ has two consecutive nonzero entries in its top row then so does $\mathcal{L}$. Thus $\mathcal{L}$ is primitive (i.e. there is some integer $n>0$ such that $\mathcal{L}^{n}>0$ ) and so $\mathcal{N}^{n}>0$ and $\mathcal{N}$ is primitive. Thus a sufficient condition for the primitivity of a nonnegative matrix is that two consecutive entries in its top row are non-zero.

The significance of the primitivity of $\mathcal{L}$ is that it enables us to draw conclusions with regard to the ergodicity of the system. In fact, if $\mathcal{L}$ is primitive then the dynamics of the total population size are governed by $\lambda_{1}$ and it is possible to completely describe the stable size distribution and the long-term behavior of the population using only $\lambda_{1}$ and its associated 
eigenvector $\vec{v}_{1}$. We now apply this theory to the system (1).

Forming the inner product of the first equation of (3) with $\vec{w}^{T}$ we obtain

$$
P(t+1)=\left(a_{0}(t) \vec{w}^{T} \mathcal{I}+a_{1}(t) \vec{w}^{T} \mathcal{L}+\cdots+a_{r}(t) \vec{w}^{T} \mathcal{L}\right) \vec{x}(t)
$$

and, noting that $\vec{w}^{T} \mathcal{L}^{k} \vec{x}(t)=\vec{w}^{T} \mathcal{L}^{k} \vec{\eta}(t) P(t)$, we have

$$
P(t+1)=\left(a_{0}(t)+a_{1}(t) \vec{w}^{T} \mathcal{L} \vec{\eta}(t)+\cdots+a_{r}(t) \vec{w}^{T} \mathcal{L}^{r} \vec{\eta}(t)\right) P(t) .
$$

By Theorem 1, we know that $\vec{w}^{T} \mathcal{L}^{k} \vec{\eta}(t) \rightarrow \vec{w}^{T} \mathcal{L}^{k} \vec{v}^{+}=\lambda^{+^{k}} \vec{w}^{T} \vec{v}^{+}=\lambda^{+^{k}}$, and thus Equation (6) is asymptotic to the limit equation

$$
P(t+1)=\left(a_{0}(t)+a_{1}(t) \lambda^{+}+\cdots+a_{r}(t) \lambda^{+^{r}}\right) P(t),
$$

a scalar equation for the weighted sum $P(t)$. It should be noted that the question of the equivalence of solutions of Equations (6) and (7) is not a trivial one and, at least in the case of aperiodic trajectories of $P(t)$, has not been completely answered. For a treatment of this issue see LaSalle [12] or Cushing [7].

\section{An Application to Population Dynamics}

We now apply the results of the preceding section to a class of size-structured competition models in which the population is structured by size-classes according to body length $s$. Many species' resource uptake rates are proportional to $s^{\tau}$ with $\tau$ between 2 and 3 (Werner and Gilliam [13], Calder [1], 
Hall et al. [10]); i.e. an individual's ability to consume food scales with body surface area or volume. Here we assume that the resource uptake rate scales with body surface area, so $\tau=2$ and

$$
P(t)=\sum_{i=1}^{m} s_{i}^{2} x_{i}(t)
$$

is the total surface area at time $t$, although a similar analysis can be carried out for $\tau=3$. Examples of species for which resource uptake scales as body surface area are such filter feeders as zooplankton, mollusks, and anemones (Hall et al. [10]). We also assume that the resource in question, denoted by $R$, is in limiting supply, and that intraspecific competition, both exploitative and possibly interference, occurs. We begin by giving a brief description of the class of models to be studied. For a more complete treatment of the derivation of these models, see Cushing [8] or Crowe [3].

Let $0<\bar{s}_{0}<\bar{s}_{1}<\cdots<\bar{s}_{m}$ define $m$ size classes $\left[\bar{s}_{j-1}, \bar{s}_{j}\right), j=1,2, \ldots, m$. Let $x_{j}(t)$ denote the number or density of individuals in the $j^{\text {th }}$ size class $\left[\bar{s}_{j-1}, \bar{s}_{j}\right)$ at time $t$, for $t=0,1,2, \ldots$, and let $\vec{x}(t)=\operatorname{col}\left(x_{i}(t)\right)_{i=1}^{m}$ be the column vector of these densities. Then the dynamics of the population are described by the matrix difference equation

$$
\vec{x}(t+1)=\mathcal{P} \vec{x}(t)
$$

where the projection matrix $\mathcal{P}$ can be decomposed into the sum of a class transition matrix $\mathcal{T}$ and a fertility matrix $\mathcal{B}$; i.e. $\mathcal{P}=\mathcal{T}+\mathcal{B}$. 
We assume that all newborns lie in the smallest size class $\left[\bar{s}_{0}, \bar{s}_{1}\right)$, that no individual can shrink in size, and that the size class intervals have lengths such that an individual can grow in length no more than into the next size class in one unit of time. Under these assumptions, the transition matrix $\mathcal{T} \geq 0$ is the bidiagonal matrix given by

$$
\pi\left[\begin{array}{ccccc}
1-\beta_{1} u(R, P) & 0 & 0 & \cdots & 0 \\
\beta_{1} u(R, P) & 1-\beta_{2} u(R, P) & 0 & \cdots & 0 \\
0 & \beta_{2} u(R, P) & 0 & \cdots & 0 \\
0 & 0 & \ddots & & \vdots \\
\vdots & \vdots & \ddots & \ddots & \vdots \\
0 & 0 & \cdots & \beta_{m-1} u(R, P) & 1
\end{array}\right]
$$

and the fertility matrix $\mathcal{B}$ is given by

$$
\mathcal{B}=\pi u(R, P)\left[\begin{array}{cccc}
\gamma_{1} & \gamma_{2} & \cdots & \gamma_{m} \\
0 & 0 & \cdots & 0 \\
\vdots & \vdots & \cdots & \vdots \\
0 & 0 & \cdots & 0
\end{array}\right]
$$

where $\pi$ is the probability that an individual survives one unit of time, $\beta_{j}$ and $\gamma_{j}$ are, respectively, the growth and reproductive coefficients for size class $j$, and $u(R, P)$ is the resource uptake rate per unit body surface area at resource level $R$ and weighted population size $P$.

Thus the dynamics of the population surface area $P(t)$ and the population area vector $\vec{x}(t)$ are governed by Equation (3) with

$$
a_{0}(t)=\pi\left(1-\beta_{q} u(R(t), P(t))\right), \quad a_{1}(t)=\pi u(R(t), P(t))
$$


where $\mathcal{L}$ is the Usher matrix

$$
\mathcal{L}=\left[\begin{array}{ccccccc}
\beta_{q}-\beta_{1}+\gamma_{1} & \gamma_{2} & \cdots & \gamma_{q} & \cdots & \gamma_{m-1} & \gamma_{m} \\
\beta_{1} & \beta_{q}-\beta_{2} & \cdots & 0 & \cdots & 0 & 0 \\
\vdots & \vdots & \ddots & \vdots & & \vdots & \vdots \\
0 & 0 & \cdots & 0 & \cdots & 0 & 0 \\
\vdots & \vdots & & \vdots & \ddots & \vdots & \vdots \\
0 & 0 & \cdots & 0 & \cdots & \beta_{q}-\beta_{m-1} & 0 \\
0 & 0 & \cdots & 0 & \cdots & \beta_{m-1} & \beta_{q}
\end{array}\right]
$$

with $\beta_{q}$ defined to be the largest $\beta_{i}$.

\subsection{Model of Resource}

We consider a resource in limited supply and denote the amount of resource available for consumption at time $t$ by $R(t)$. We assume that there is a constant amount of resource $I_{0}$ input each unit of time and that the resource not consumed over the course of one unit of time is subject to a non-consumptive decrease or loss, for example through degradation or through a washout process. Thus the amount of resource available at time $t+1$ is given by

$$
R(t+1)=\xi\left[\begin{array}{c}
\text { amount of resource } \\
\text { remaining after consumption }
\end{array}\right]+I_{0}
$$

where $\xi \in[0,1)$ is the fraction of unconsumed resource remaining after one unit of time.

Let $r(R)$ be the inherent resource uptake rate per unit body surface area of consumer at resource level $R$ and let $1-c(P)$ be the fractional decrease in the uptake rate due to the presence of $P$ competitors. We assume

$$
\begin{array}{ll}
\text { [A1] } & r(0)=0, \quad r^{\prime}(R)>0, \quad \lim _{R \rightarrow+\infty} r(R)=r^{0}<+\infty \\
\text { [A2] } & c(0)=1, \quad c^{\prime}(P) \leq 0, \quad c(P) \leq 1 \text { for } P>0 .
\end{array}
$$


An example of such an $r$ is the Michaelis-Menten uptake function $r(R)=$ $\frac{m R}{a+R}$ with $m, a>0$. The density term $c(P)$ can also be modeled in various ways ranging from purely exploitative competition $(c(P) \equiv 1)$ to strong nonlinear effects (e.g. $c(P)=e^{-d P}, d>0$ ). In the models we consider, if $c(P) \not \equiv 1$ we will assume that $c(P)<1$ for $P>0$ and that $c^{\prime}(P)<0$. As one example of such a function, let $1-e^{-d}(d>0)$ be the fractional decrease in resource uptake of an individual per unit body surface area of a competitor. Then at time $t$, a fraction $e^{-d P(t)}$ of the per unit inherent uptake $r(R(t))$ is actually consumed. Note that if $d=0$ we have the purely exploitative case $c(P) \equiv 1$, and larger values of $d$ result in stronger competitive or density effects.

Then the resource uptake rate per unit body surface area at time $t$ becomes $u(R(t), P(t))=r(R(t)) c(P(t))$ as long as the total amount of resource consumed does not exceed $R(t)$; i.e. as long as $R(t) \geq r(R(t)) c(P(t)) P(t)$. Since the amount of resource available at time $t$ is $R(t)$, the maximum resource consumption per unit body surface area is $\frac{R(t)}{P(t)}$.

Thus we have the following model of the dynamics of the resource $R$ :

$$
R(t+1)=\xi[R(t)-u(R(t), P(t)) P(t)]+I_{0}
$$

where

$$
u(R, P)=\left\{\begin{array}{cl}
r(R) c(P) & \text { if } R \geq r(R) c(P) P \\
\frac{R}{P} & \text { if } R<r(R) c(P) P
\end{array}\right.
$$




\subsection{Preliminary Results}

Clearly $\mathcal{L} \geq 0$ and since the $\beta_{i}$ are chosen so that $1-\beta_{i} u(R, P)>0$, we know that there exists an $a_{0}$ such that $0 \leq a(t) \leq a_{0}=\pi$. Thus in order to apply Theorem 1 we need only show that $b(t)$ is bounded away from 0 . To this end we consider the boundedness of $P(t)$ and $R(t)$. The following results are stated without proof; proofs may be found in Crowe [3].

Theorem 2 If $\vec{x}(t)$ is a solution of Equation (8) with $\mathcal{T}$ and $\mathcal{B}$ given by Equations (9) and (10) where $c(P) \not \equiv 1$ with $\vec{x}(0) \geq \overrightarrow{0}(\neq \overrightarrow{0})$, then $\vec{x}(t) \geq \overrightarrow{0}$, $P(t)>0$, and both sequences are bounded for all $t>0$.

Theorem 3 If $R(t)$ is a solution of.Equation (11) with $u(R, P)$ as defined previously and with $r(R)$ and $c(P)$ satisfying assumptions [A1] and [A2] respectively, then for $R(0) \geq 0$ we have $R(t) \geq I_{0}$ for $t>0$ and $R(t)$ bounded above.

If we let $b_{0}=\pi r\left(I_{0}\right) c\left(P^{\infty}\right)$ where $P^{\infty}$ is an upper bound for $P(t)$, we have $0<b_{0} \leq b(t)$ for all $t \geq 0$. Now as long as $\mathcal{L}$ satisfies $\mathbf{H 2}$ of Theorem 1 (e.g. species has two consecutive reproducing classes), the dynamics of $P(t)$ are determined by the scalar difference equation

$$
P(t+1)=\pi(1+\theta u(R(t), P(t))) P(t)
$$

with $P(0)=\vec{w}^{T} \vec{x}(0)>0$ where $\theta \equiv \lambda^{+}-\beta_{q}$. Since $\lambda^{+}>0$ is the dominant eigenvalue of $\mathcal{L}, \theta$ is the eigenvalue of the matrix 


$$
\mathcal{M} \equiv \mathcal{L}-\beta_{q} \mathcal{I}=\left[\begin{array}{ccccc}
-\beta_{1}+\gamma_{1} & \gamma_{2} & \cdots & \gamma_{m-1} & \gamma_{m} \\
\beta_{1} & -\beta_{2} & \cdots & 0 & 0 \\
0 & \beta_{2} & \ddots & \vdots & \vdots \\
\vdots & \vdots & \ddots & -\beta_{m-1} & 0 \\
0 & 0 & \cdots & \beta_{m-1} & 0
\end{array}\right]
$$

with largest real part. Thus we may study the dynamics of the $n+1$ dimensional system given by Equations (3) and (11) by considering instead the 2-dimensional system given by Equations (12) and (11). More details and result: can be found in Crowe [5] and Cushing [6],[7]. We now illustrate this approach numerically with a 3 -size class example.

\subsection{Numerical Example}

To illustrate the results presented above, we study a 3 -size class model of the form (8) with $\pi=.5, d=0.1, I_{0}=10, \beta_{1}=0.99, \beta_{2}=0.95$, and $\gamma_{1}=0$. We study the dynamics of the three size classes, $x_{1}, x_{2}, x_{3}$, and the total population size, $P$, and compare these results with those predicted by the theory. We give four examples, showing total population density (1) approaching zero (extinction), (2) equilibrating to a positive value, (3) approaching a 2-cycle, and (4) exhibiting aperiodic dynamics. In each case the time series of $P$ is shown along with a graph of the normalized size class distribution. In addition, the dominant eigenvalue and correesponding eigenvector used in the Theorem 1 are stated for comparison purposes. For these examples, $\mathcal{L}$ is given by 


$$
\mathcal{L}=\left[\begin{array}{ccc}
0 & \gamma_{2} & \gamma_{3} \\
.99 & .04 & 0 \\
0 & .95 & .99
\end{array}\right]
$$

where $\gamma_{2}$ and $\gamma_{3}$, the birth rates of the second and third size classes, are the parameters that are varied in each case to cause the changes in the dynamics of $P$.

\section{Discussion}

In this paper we have presented an ergodic theorem for a general class of nonlinear systems. This theorem proves the existence of a so-called limiting vector and shows that all (vector) solutions of the system must approach in direction that limiting vector, regardless of the magnitude of the solution. This allows us to study the dynamics of an aggregate variable without losing information about the behavior of elements of the vector solution. In fact, since the limiting equation (7) involves the parameter $\lambda^{+}$which is an eigenvalue of a shift of $\mathcal{L}$, it is possible to relate the dynamics of the aggregate variable $P(t)$ to the values of parameters in the original system. Examples of this analysis may be found in Caswell [2] and Crowe [3],[4].

We have illustrated the ergodic theorem with a 3 size-class model of competition for a dynamically modeled resource. Such models have been studied assuming constant resource levels and the diagonalizability of $\mathcal{L}$, but the theorem presented in this paper allows us to remove both of these assumptions. 
In studying the dynamics of the limiting equation we find the existence of a nontrivial equilibrium and see the bifurcation of this equilibrium to a 2-cycle, results which have enabled us to carry on multispecies competition studies of similar systems (see Crowe [5]).

The key remaining mathematical questions concern the relationship between invariant sets of the original equation for $P(t)$ and the limiting equation. In addition, many biological generalizations of the given model may be posited to study a variety of interesting competitive interactions such as predator-mediated competition and asymmetric competitive effects.

In the case of many applications of nonlinear equations, the level of complexity of a physically realistic model makes mathematical analysis of the model a formidable task. The ergodic theorem presented here provides one way of simplifying some detailed models to facilitate analysis without losing the details in the process. 


\section{CAPTIONS}

FIG. $1 \gamma_{2}=1.0, \gamma_{3}=1.0$. Population goes extinct $(P(t) \rightarrow 0)$. Eigenvector $\vec{v}=\left[\begin{array}{lll}0.81 & .206 & .713\end{array}\right]^{T}$ corresponding to the dominant eigenvalue $\lambda=$ 1.606 of $\mathcal{L}$.

FIG. $2 \gamma_{2}=1000, \gamma_{3}=1000$. Total population size equilibrates. Eigenvector $\vec{v}=\left[\begin{array}{lll}.883 & .109 & .008\end{array}\right]^{T}$ corresponding to the dominant eigenvalue $\lambda=31.963$ of $\mathcal{L}$.

FIG. $3 \gamma_{2}=10000, \gamma_{3}=1000$. Total population size approaches a 2-cycle. Eigenvector $\vec{v}=\left[\begin{array}{lll}.961 & .038 & .001\end{array}\right]^{T}$ corresponding to the dominant eigenvalue $\lambda=99.567$ of $\mathcal{L}$.

FIG. $4 \gamma_{2}=90000, \gamma_{3}=1000$. Total population size exhibits aperiodic dynamics. Eigenvector $\vec{v}=\left[\begin{array}{lll}.987 & .0131 & .00009\end{array}\right]^{T}$ corresponding to the dominant eigenvalue $\lambda=298.5$ of $\mathcal{L}$. 


\section{References}

[1] William A. Calder III. Size, Function and Life History. Harvard University Press, Cambridge, Massachusetts, USA, 1984.

[2] H. Caswell. Matrix Population Models. Sinauer Associates Inc., Sunderland, MA, 1989.

[3] K. M. Crowe. A Discrete Size-Structured Competition Model. PhD thesis, University of Arizona, Tucson, Arizona, 1991.

[4] K. M. Crowe. Parameter dependence, size, and survivability. Technical Report !!!!!!, Biometrics Unit, Cornell University, 1992.

[5] K. M. Crowe. Size-structured competitive coexistence in discrete time. Technical Report !!!!!!, Biometrics Unit, Cornell University, 1992.

[6] J. M. Cushing. Nonlinear matrix models and population dynamics. Natural Resource Modeling, 2(4), 1988.

[7] J. M. Cushing. A Strong Ergodic Theorem for some nonlinear matrix models for structured population growth. Natural Resource Modeling, 3(3):331-357, 1989.

[8] J. M. Cushing. Some competition models for size-structured populations. Rocky Mountain Journal of Mathematics, 20(4), 1990. 
[9] F. R. Gantmacher. Applications of the Theory of Matrices. Interscience Publishers, New York, 1959.

[10] Donald J. Hall, Stephen T. Threlkeld, Carolyn W. Burns, and Philip H. Crowley. The Size-Efficiency Hypothesis and the structure of zooplankton communities. Ann. Rev. Ecol. Syst., 7:177-208, 1976.

[11] J. Impagliazzo. Deterministic Aspects of Mathematical Demography, volume 13 of Biomathematics. Springer, Berlin, 1980.

[12] J. P. LaSalle. The Stability of Dynamical Systems. Reg. Conf. Series in Appl. Math. SIAM, Philadelphia, 1976.

[13] E. Werner and J.F. Gilliam. The ontogenetic niche and species interactions in size-structured populations. Ann. Rev. Ecol. Syst., 15:393-425, 1984. 
Fig. 1 a

Normalized Size Class Distribution

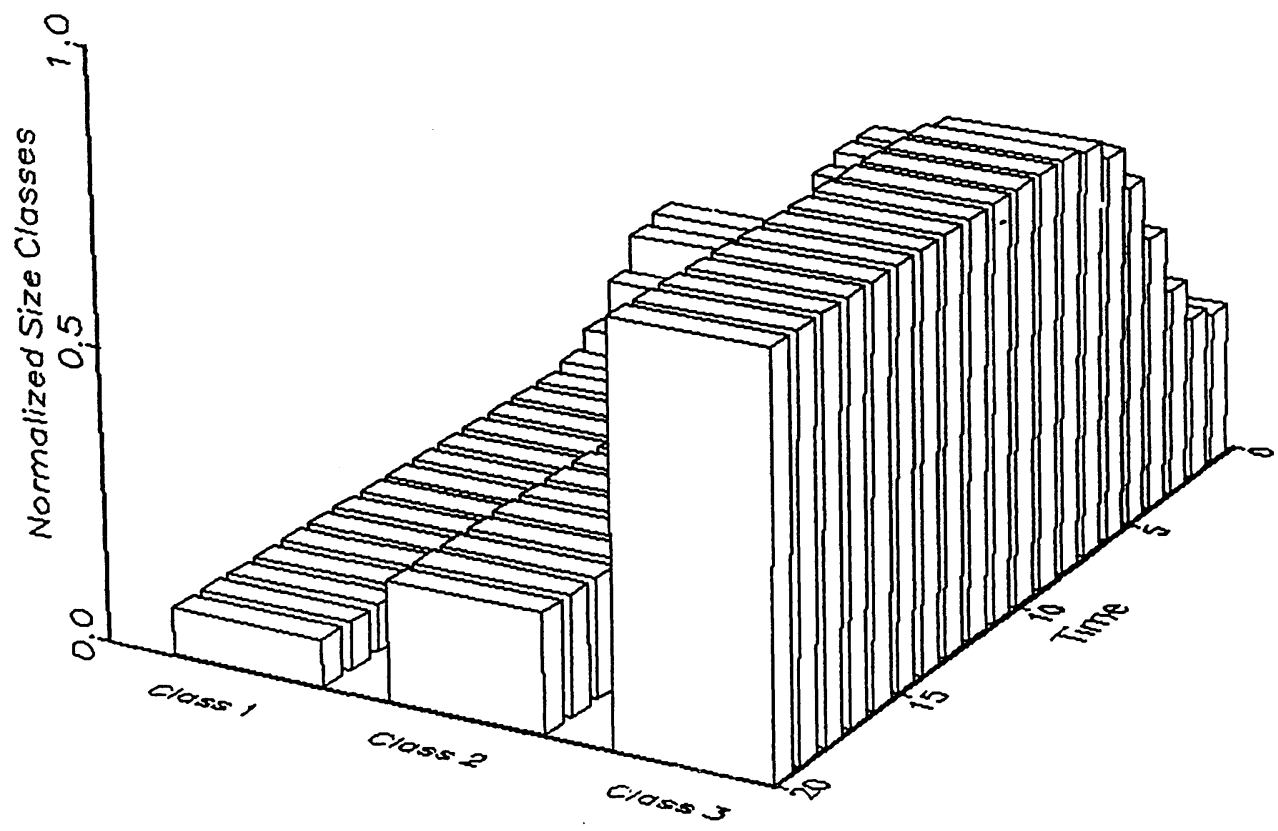

Fig. $1 \mathrm{~b}$

Weighted Population Size

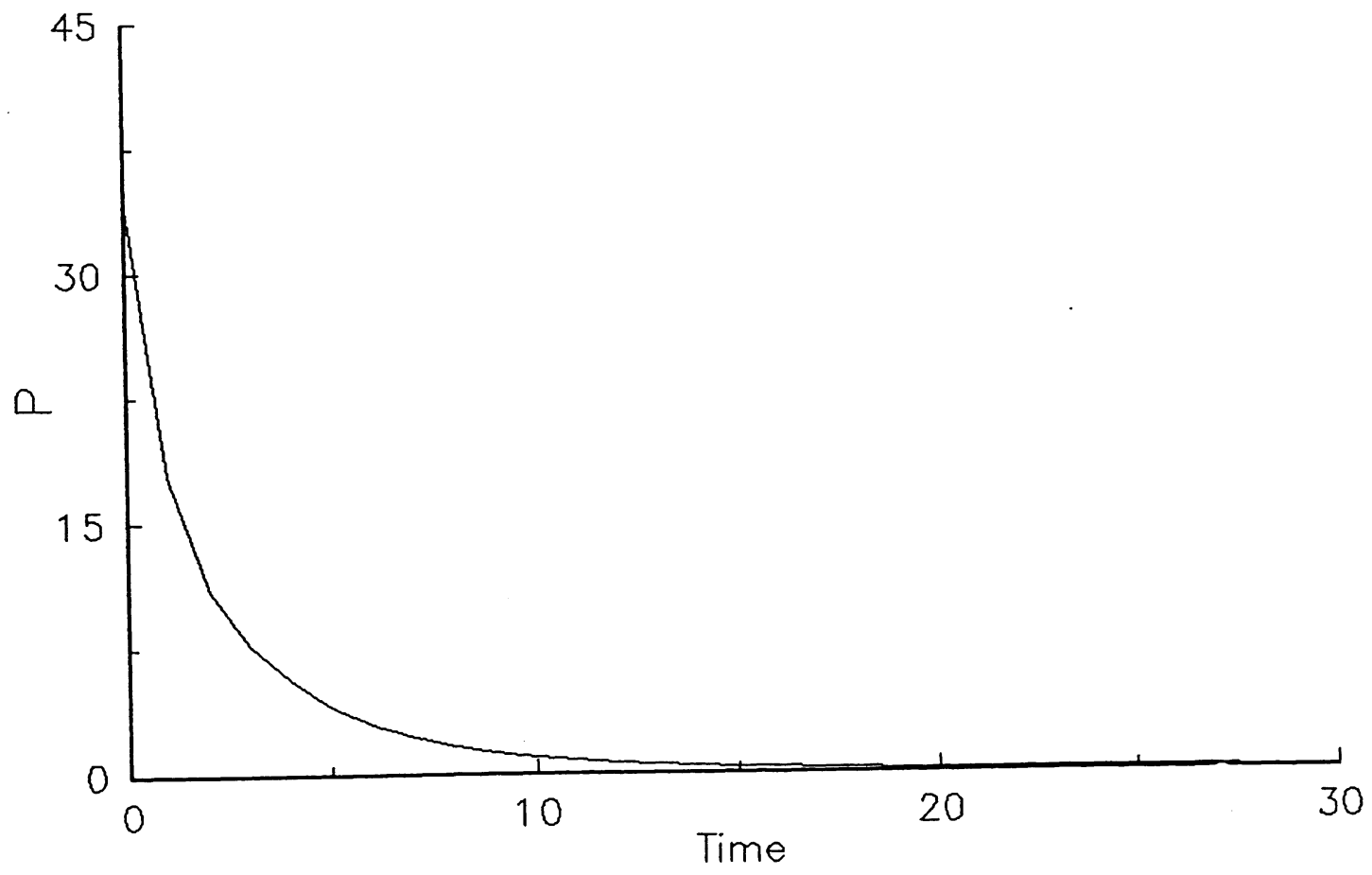


Fig. $2 a$

Normalized Size Class Distribution

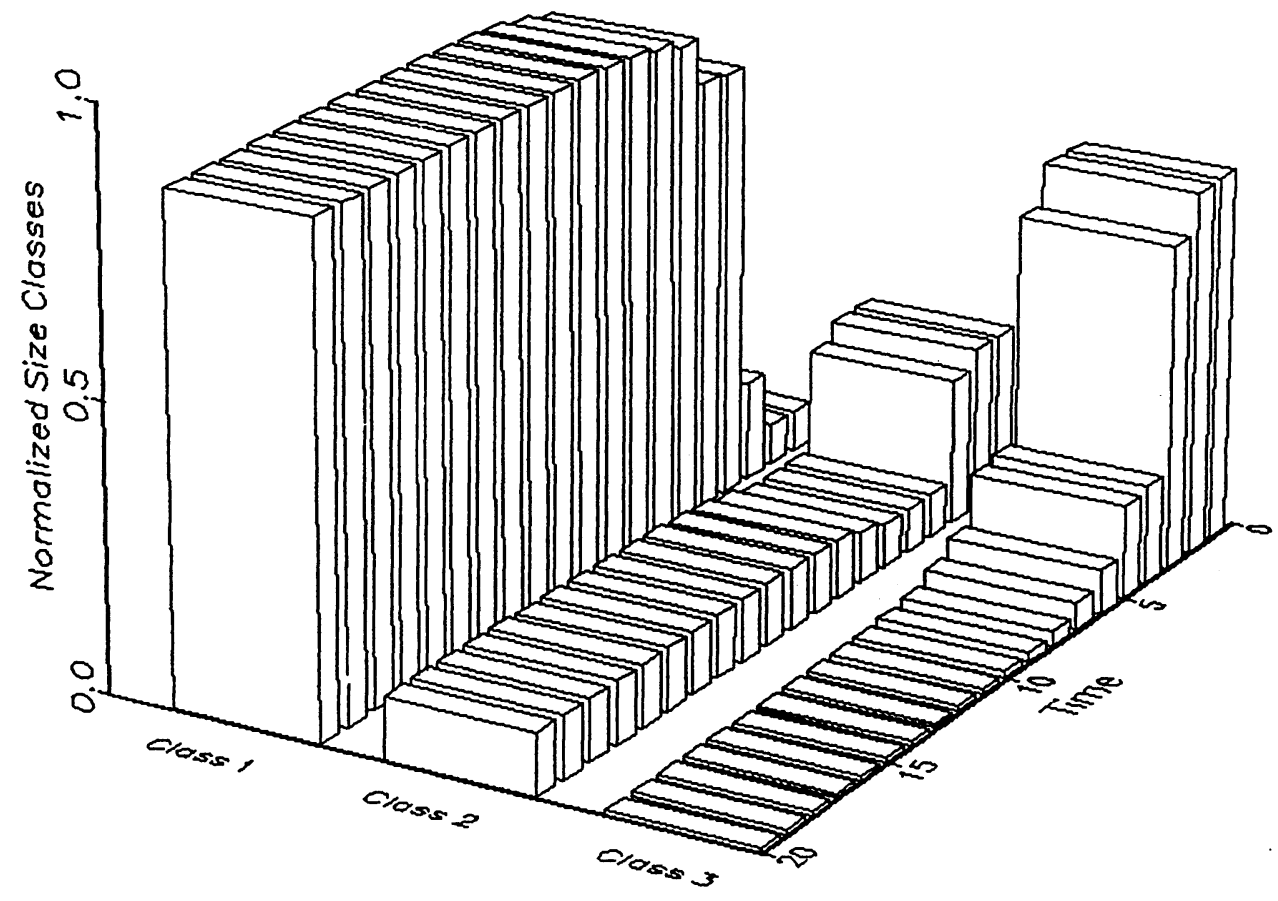

Fig. 2b

Weighted Population Size

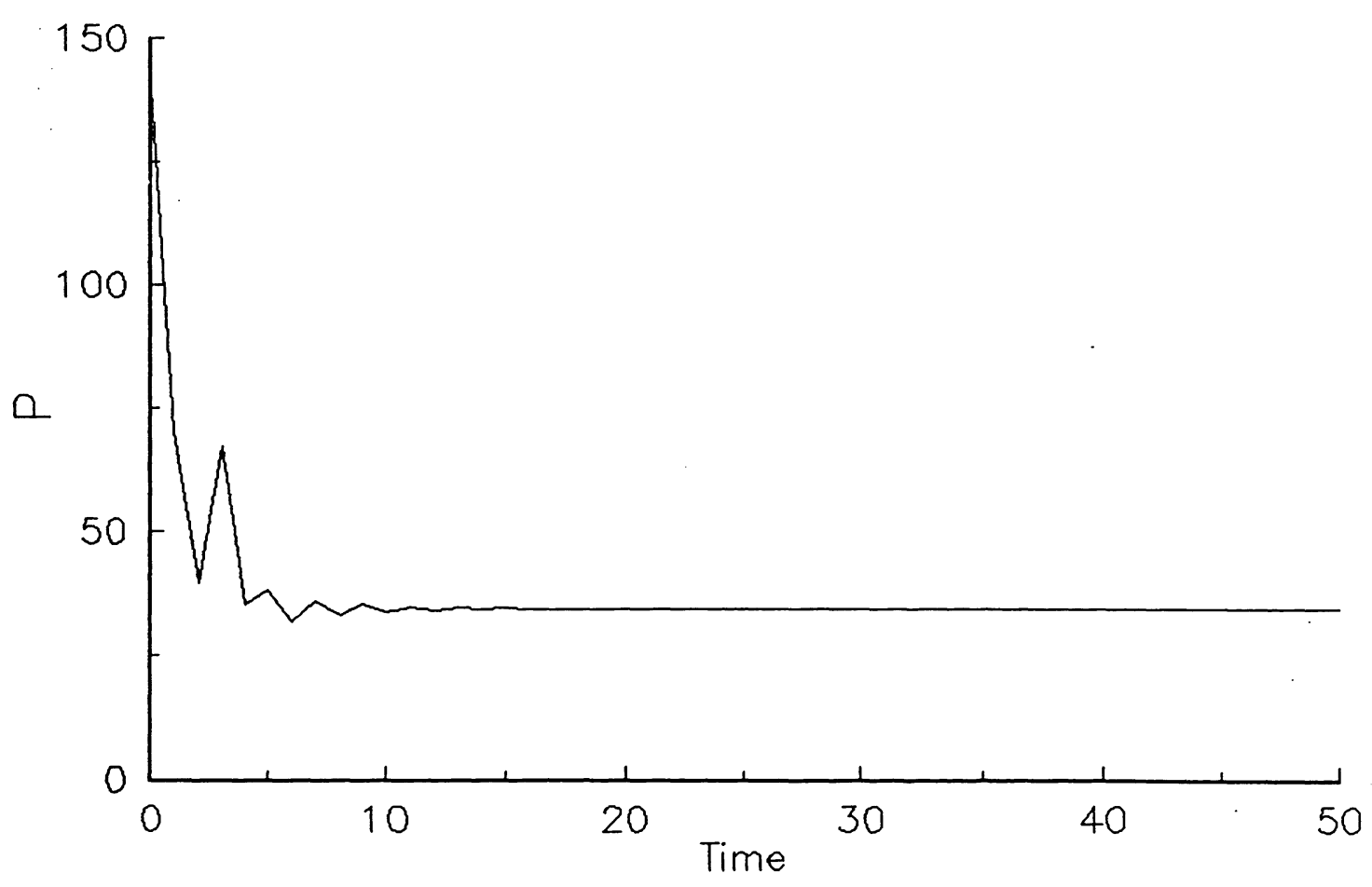


Fig. 3a

Normalized Size Class Distribution

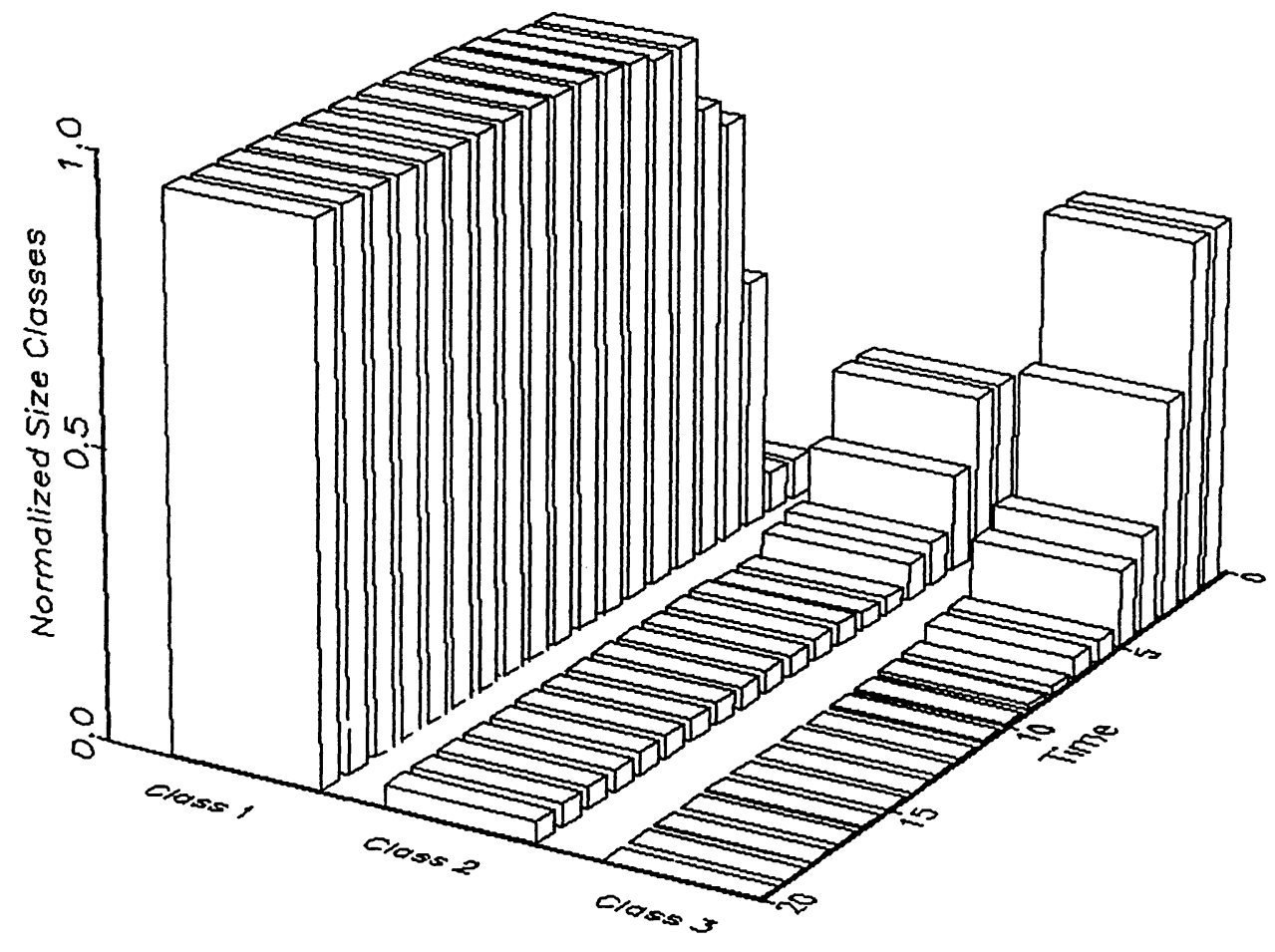

Fig. 3b

Weighted Population Size

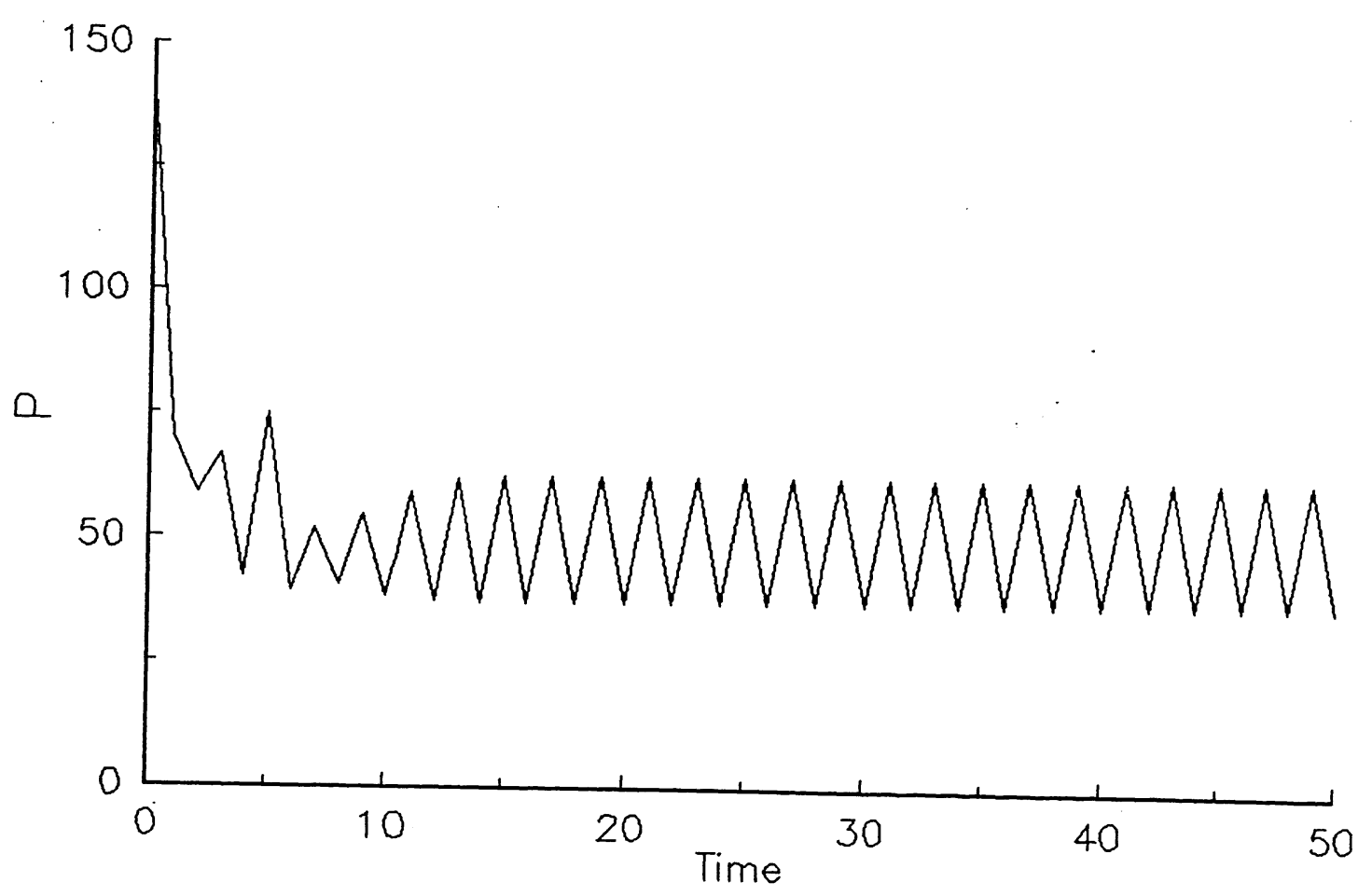


Fig. 4a

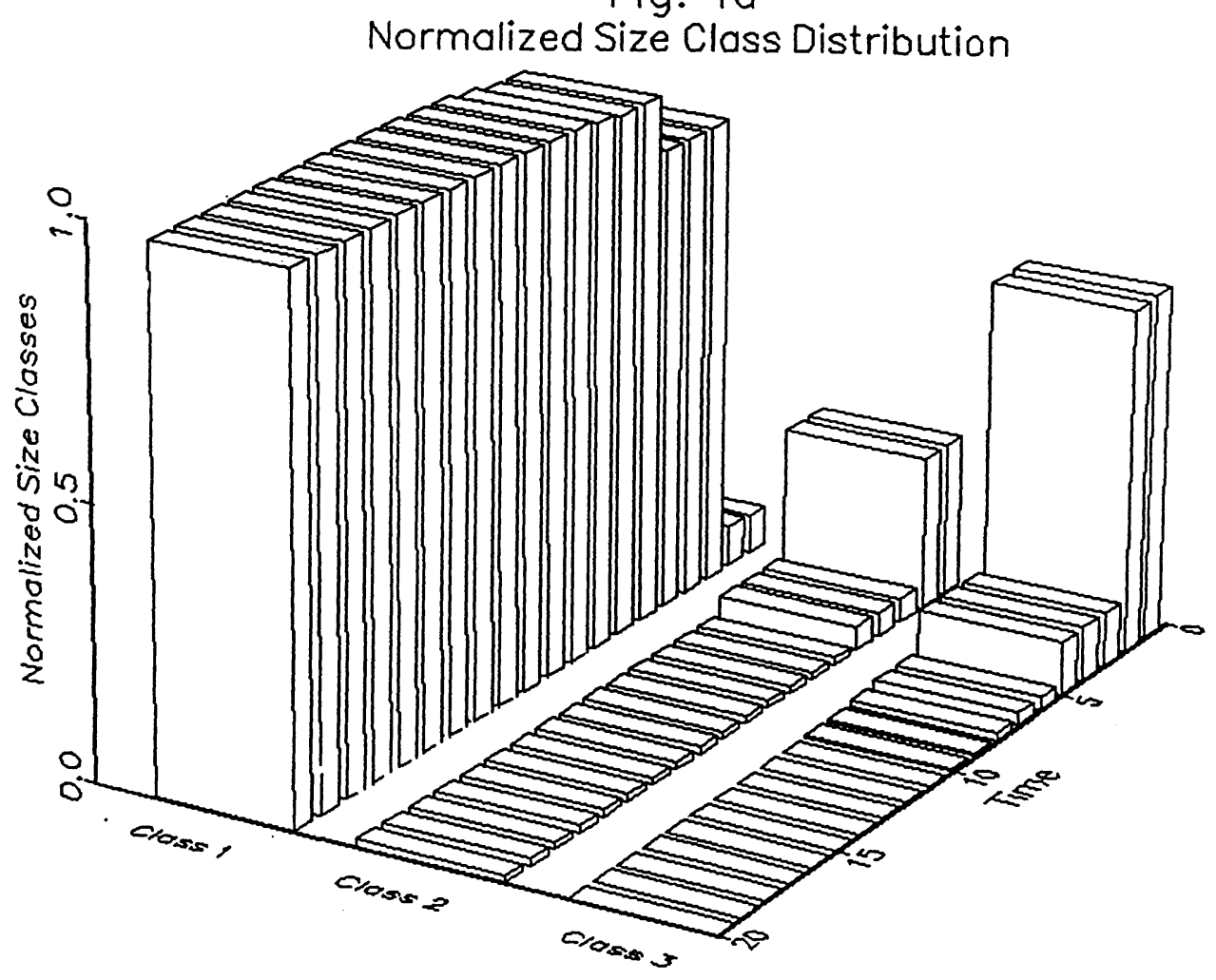

Fig. 4b

Weighted Population Size

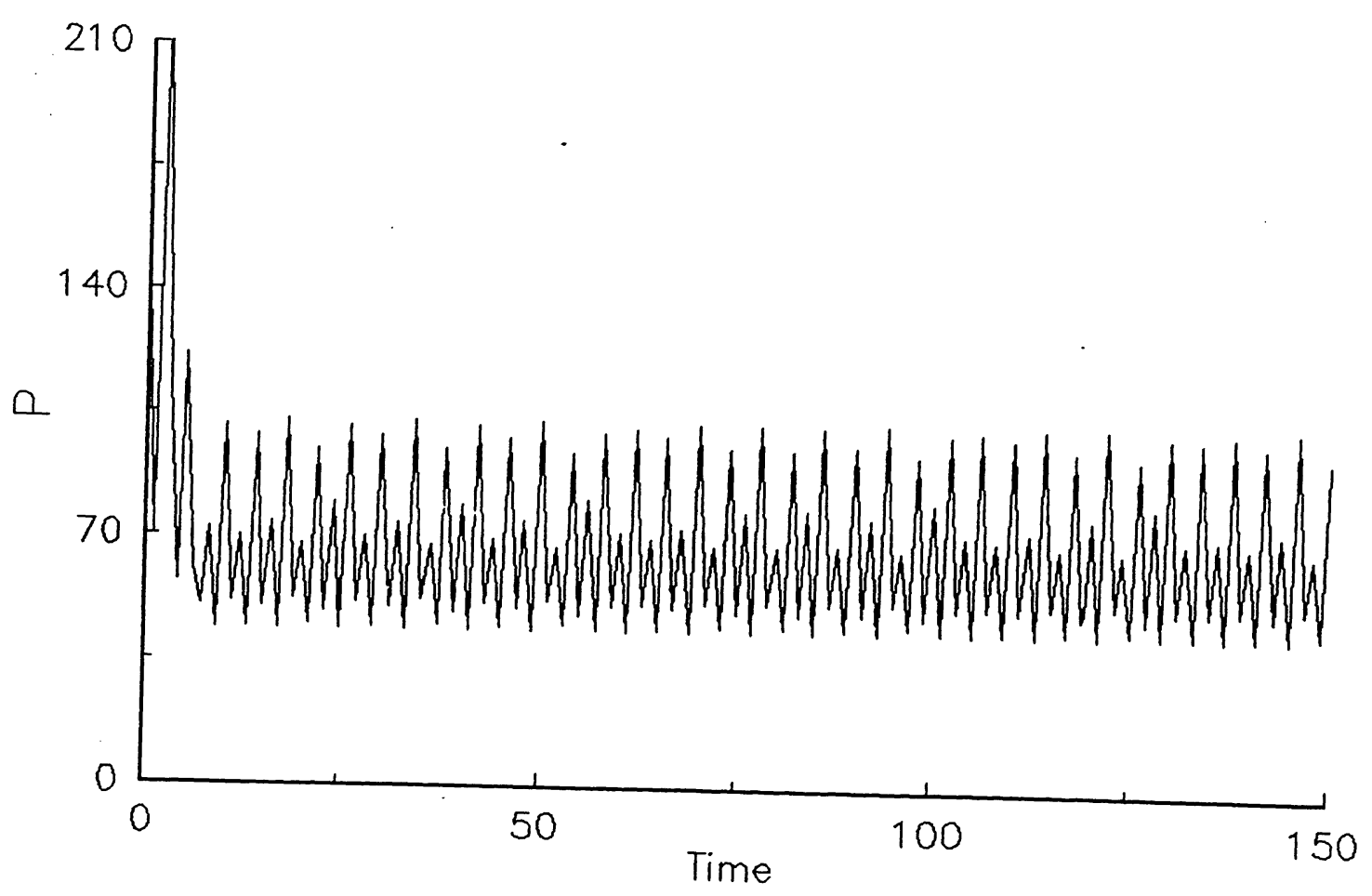

